\title{
Technoeconomic Assessment Based on Active Context-Knowledge Orchestration for Power Internet of Things
}

\author{
Yuanshuo Zheng $\mathbb{D},{ }^{1}$ Shujuan Sun, ${ }^{1}$ Chenyang Li, ${ }^{1}$ Jingtang Luo $\mathbb{D},{ }^{2}$ Jiuling Dong, \\ Yudong Wang, ${ }^{3}$ and Xiaolong Yang $\mathbb{D}^{1}$ \\ ${ }^{1}$ School of Computer and Communication Engineering, University of Science and Technology Beijing, \\ Haidian District, Beijing 100083, China \\ ${ }^{2}$ State Grid Sichuan Economic Research Institute, Wuhou District, Chengdu 610041, China \\ ${ }^{3}$ State Grid Economic and Technological Research Institute Co., Ltd, Changping District, Beijing 102209, China
}

Correspondence should be addressed to Jingtang Luo; 406996203@qq.com

Received 30 July 2021; Accepted 18 October 2021; Published 31 October 2021

Academic Editor: Xin Liu

Copyright (c) 2021 Yuanshuo Zheng et al. This is an open access article distributed under the Creative Commons Attribution License, which permits unrestricted use, distribution, and reproduction in any medium, provided the original work is properly cited.

\begin{abstract}
Power Internet of Things (abbreviated as PIoT) is the information infrastructure to provide ubiquitous perception ability for smart grid (abbreviated as SG). To better deploy and utilize PIoT, its perception ability must be comprehensively assessed in terms of technical performance and economic benefits. However, at present, there is no assessment framework for PIoT due to the high diversity and heterogeneousness of SG scenarios. Additionally, there is information overlap between metrics in the assessment framework. The assessment model which could remove redundant information between metrics and simplify the assessment framework is an urgent demand to improve the effectiveness and timeliness of assessment. Consequently, first, aiming at the power system requirements of complex and diverse, a general assessment framework is put forward to assess the ability of PIoT in terms of technology and economy. Next, the requirement characteristics of power distribution scenario (abbreviated as PDS) are precisely analyzed with active context-knowledge orchestration technology. The general assessment framework is instantiated to build an instantiation assessment scheme in PDS. Moreover, an assessment model is established based on the instantiation assessment scheme to assess the efficiency of PIoT in Beijing. Finally, the assessment model is further refined with the machine learning technology to improve the efficiency of assessment. This refinement model achieves the extraction of 4-dimensional metrics from 23-dimensional metrics for assessment and finally improves assessment efficiency by $82.6 \%$.
\end{abstract}

\section{Introduction}

As being a hub of the energy system, smart grid (abbreviated as SG) has the important mission that it not only unblocks the energy industry chain but also promotes the achievement of carbon peaks and carbon neutrality goals [1]. Consequently, it is necessary to continuously strengthen the integration of power grid (abbreviated as PG), information network, and energy network to form a new energy ecosystem with SG as the main body $[2,3]$. Meanwhile, with the continuous upgrading and transformation of the power system, the Internet of Things (abbreviated as IoT) plays an increasingly important role in it [4]. The IoT is a network form that uses information sensing equipment to connect objects and the Internet to communicate in accordance with an agreed protocol to achieve intelligent tracking and management of objects. As a research hotspot in academia and industry, it has attracted widespread attention. For instance, Xin and Zhang studied the resource allocation of the Cognitive Industrial Internet of Things (abbreviated as CIIoT) and proposed a cluster-based CIIoT to improve sensing and transmission performance of the CIIoT [5]. Additionally, in order to improve the transmission performance, Liu et al. also proposed a multibeam satellite Industrial Internet of Things (abbreviated as IIoT) with nonorthogonal multiple access (abbreviated as NOMA), 
while guaranteeing the QoS for the satellite IIoT, including the required transmission rate and transmission delay [6]. The IoT combines communications, information, sensing, automation, and other technologies. It widely deploys various intelligent sensing devices with certain perception, computing power, and execution capabilities in all aspects of power production, transportation, consumption, and management. Importing IoT technology to power system can effectively integrate the communication infrastructure resources and power system infrastructure resources, which can improve the information level of power system and the utilization efficiency of the existing infrastructure and provide important technical support for power generation, transmission, transformation, and distribution [7]. The Power Internet of Things (abbreviated as PIoT) is a network system formed by connecting all objects related to power [8]. It is the specific manifestation of the IoT in the power industry as well as the infrastructure for SG to achieve information interaction [9]. In power systems, communication between a large number of devices required the need for greater capacity, greater connectivity, and higher data rates which can be achieved by merging $5 \mathrm{G}$ with IoT. This is because the $5 \mathrm{G}$ cellular network has promising features to provide much lower latency, increased integration within the mobile network, and longer range [10]. The PIoT uses widely deployed terminal to collect massive data, which can provide data support for the organic fusion of PG, information network, and energy network $[11,12]$. PIoT is made up of various IoT sensors, smart terminals, cloud platforms, and other devices with collecting massive data connected through various communication networks [13]. Analyzing and processing of collected data, the overall operation law of the SG can be discovered with the data relationship, which can improve the visibility of the SG [14]. Accordingly, the visibility and controllability of the PIoT will affect the operation security of SG.

The efficiency assessment for PIoT is a significant way to measure the development of PIoT. However, there is a lack of relevant work about the efficiency assessment of PIoT. The study in related fields can provide research ideas, such as the SG assessment and the Energy Internet assessment [15-19].

In the field of SG assessment, Li designed an evaluation model integrating TOPSIS and grey management analysis method, which is successfully applied to evaluating the development of economic benefit in five service areas of power supply in Zhejiang province [20]. Hall and Foxon applied the cost-benefit method to quantitatively analyze the economic benefits of the SG in a certain area of the United Kingdom [21]. However, the above research studies only analyzed the economic benefit of the SG, neglected the multiattribute problem of the SG from the technical, environmental, and other aspects, and failed to build a multilevel evaluation model to comprehensively evaluate the SG. Unlike them, Zeng et al. built a modified comprehensive evaluation index system of demand response benefit covering economy and environment to provide effective guidance for scientific investment decision-making of the intelligent distribution network [22]. Li and Chen designed an indicator system to evaluate the potential for regional electric energy substitution in China from the aspects of socioeconomic level, energy consumption structure, and environmental protection constraints [23]. Next, the TOPSIS method with improved connection degree was used to comprehensively evaluate the regional power substitution potential.

SG is the core of the Energy Internet, which is the grid with distributed, open and shared based on renewable energy. In the area of Energy Internet efficiency evaluation, Fang et al. presented an evaluation index system containing intermittent energy indicators based on the analysis of influential factors of operation and economic efficiency of the power system [24]. Nevertheless, this study only evaluated the PG from two aspects of operating efficiency and investment benefits, which made the research perspective slightly single. $\mathrm{Lu}$ et al. constructed a novel evaluation criteria system from the economic aspect, environmental aspect, technical aspect, energy aspect, and service aspect [25]. This system can make assessment comprehensive and objective for energy management system of regional integrated. However, literature [24] and literature [25] did not consider the demand differences of research subjects in different power system scenario (abbreviated as PSS) when evaluating research subjects. Liu et al. proposed a unified evaluation model to assess the adjustable capability of distributed energy systems under typical scenarios at a single moment, and the impact of economic constraints on the adjustable capability was also extensively analyzed [26].

The abovementioned works generally assess the object of study from economic and technical, which can provide a useful reference for the subsequent assessment of PIoT. Nevertheless, existing research studies on PIoT are rare, and there are still some problems which are needed to be solved. The main problems are listed as follows:

(1) There lacks a general assessment framework for PIoT. PIoT is the basis for SG to collect data. Analyzing massive data, the operation rules and potential risks of the SG can be found, which can achieve stable operation and optimal allocation of resources for the power system. Accordingly, we need to assess whether the efficiency of PIoT meets the requirement for SG development.

(2) There lacks an instantiation assessment scheme for PIoT in typical scenario. The types of PIoT nodes in different PSS are different, and the perception requirements are complex and diverse. Consequently, the requirement abilities of PIoT differ in different PSS. Hence, the assessment framework should be specific to certain scenarios.

(3) There lacks a dimensionality reduction model for instantiation assessment scheme. The phenomenon of information intersection will inevitably occur among the indexes of the instantiation assessment scheme. Accordingly, the main features of the instantiation assessment scheme are extracted by using the dimensionality reduction model, which can 
reduce the dimensionality of the instantiation assessment scheme. It weakens the complexity of the assessment model and improves timeliness of the assessment.

To fill these gaps mentioned above, this paper closely integrates the current internal environmental factors such as the requirement and the development direction of the PG and external environmental factors such as technological development and national policies, which were faced by the current development of PIoT. Additionally, the technical route is adopted in this paper: first, building a general assessment framework; next, reshaping the instantiation assessment scheme in certain scenarios; then, establishing an assessment model; finally, achieving the comprehensive assessment and refinement analysis of PIoT.

The main contributions of this study are as follows:

(1) To comprehensively assess the efficiency of PIoT in terms of ubiquitous perception, security protection, etc., the general assessment framework of PIoT is built

(2) The core requirements of the power distribution scenario (abbreviated as PDS) are explored by using Active Context-Knowledge Orchestration Technology (abbreviated as ACKOT), and an instantiation assessment scheme with the requirement characteristics of the PDS is built

(3) The assessment model based on PCA and AHP is designed to assess the efficiency of PIoT

(4) A refinement model based on machine learning technology (abbreviated as MLT) is proposed to reduce redundant information between metrics to achieve dimensionality reduction of the instantiation assessment scheme

\section{Building an Assessment Framework}

With the in-depth development of SG, the number of functional subjects and data scale of SG are increasing rapidly. As the basis and link, PIoT will play a more prominent role in the aspects of ubiquitous perception, efficient processing of information, convenient and flexible application, and so on. Consequently, this paper refers to some standards such as the GB/T36468-2018 system evaluation index system of Internet of Things and GB/T370252018 information security technology-security technical requirements of data transmission for Internet of Things to build an assessment framework of PIoT [27-29].

2.1. Technical Index. Since there are many types of terminals in the links of transmission, transformation, and distribution and so on, such as data transfer unit, laser ranging sensor, and smart meter, the operation and maintenance efficiency of the equipment are reduced. It is particularly important to implement scientific maintenance management of the terminal. Moreover, the collection and analysis of the massive data are realized through widely distributed terminals, which can provide early warning of hidden dangers to avoid failure of PG equipment. In the meantime, different power services and power scenarios have diversified requirements for the QoS of PIoT, and some important control services have strict requirements for QoS, such as relay protection and dispatching control. Thus, we need to measure the ability of PIoT's QoS to meet application requirements. Additionally, since the data collected by the terminal contains user privacy and corporate information, and its forms are complex and diverse, the security protection of the data should be taken seriously. Consequently, the ability of PIoT is assessed to meet the requirements of the power system from ubiquitous perception, applications' QoS guarantee, security protection, and maintenance management.

The maintenance management ability is used to assess the stable operation level of the terminal. It can provide prerequisites for achieving the ubiquitous perception of PIoT. Additionally, the applications' QoS guarantee obtains massive data through the ubiquitous perception ability to meet the diverse needs of the power system. Meanwhile, security protection ability is used to assess the data security during transmission and sharing, which can provide a basic guarantee for data security.

2.1.1. Ubiquitous Perception Ability. The ability of a ubiquitous perception is using widely deployed sensing devices to obtain massive data. Hence, we intend to comprehensively assess the ubiquitous perception ability from terminal deployment coverage, rate of successful data collection, variety of terminal perception frequency, accuracy of data collection, and diversity of sensing data.

By analyzing and processing of the data, the visibility of the device status and management status in the SG can be improved. Collecting data relies on terminal equipment. Here, the terminal deployment coverage is used to measure the coverage fraction of terminal in PIoT. Meanwhile, aiming at the diversity and heterogeneity of perception data, diversity of sensing data is used to measure the diversity of massive data. Additionally, the variety of terminal perception frequency is used to measure the degree of intelligence of the terminal setting perception frequency according to the location, time, and situation. For the collection of massive data, the rate of successful data collection is used to measure the ability of PIoT successfully collecting perceptual data. Meanwhile, accuracy of data collection is used to measure the closeness between the collected data information and the real data information.

2.1.2. Applications' QoS Guarantee. PIoT is the basic information infrastructure of SG to meet the application requirement of every link from power generation to distribution. Thus, we intend to consider the applications' QoS guarantee of PIoT from the ability of data sharing, reliability for meeting application requirements, communication quality of service, and quality of data processing. It can respond to the diverse needs in the power system of massive data transmission, Internet of Everything, flexible response, and extensive perception. 
Among them, the ability of data sharing can be used to assess the ability of PIoT to meet the requirements of massive data transmission in the power system. Reliability for meeting application requirements is used to measure the ability of terminal in PIoT to always meet the application requirements in SG. The flexible compatibility of PIoT is the basis for realizing Internet of Everything in the power system. Communication quality of service is used to measure the communication effect of PIoT. For example, there are many scenarios of cooperative control in the power system. In these scenarios, power travels at the speed of light, and the end-to-end delay is less than $10 \mathrm{~ms}$ which can provide support for the power system to respond flexibly to various changes. Quality of data processing is used to measure the degree which data processing technology meets application requirement. For example, in recent years, the Edge TPU chip developed by Google has the capability of massive data processing and edge computing, which will improve the extensive perception ability of the terminal.

2.1.3. Security Protection Ability. For SG, its core is the application of massive data in power system. Hence, the security and privacy of data are particularly important. However, at present, the data interaction methods and sharing behaviors between the entities of the PG lack security guarantee, which weaken the ability of data security sharing and circulation in the entire PG. There are some security risks faced by PIoT, specifically the environmental security of data, security protection of data, and preventing the terminal from extreme disasters.

Firstly, the environmental security protection of data includes user environmental security protection and transmission environmental security protection. On the one hand, user electricity consumption data are related to user privacy. Thus, we need to ensure the privacy security of different participants when we share the data. Based on this situation, we use security and robustness to assess the security performance of the user environment. On the other hand, the extensive perception also brings challenges to communication security. Ensuring the integrity and confidentiality of data during transmission is a core issue. Accordingly, the ability of anti-interference for communication network is used to measure the security of the data transmission environment. Secondly, in view of the security protection of the data, we intend to use the ability of preventing data leakage to assess the performances for antiattack and antitheft of the data. Finally, we also need to focus on the issue of how PIoT can effectively defend against extreme disasters. Since data collection relies on various terminals, it is essential to ensure the normal operation of device in extreme disaster situation. Consequently, the ability of resisting natural is used to measure the defense ability of PIoT in response to extreme disasters.

2.1.4. Maintenance Management Ability. The maintenance management is manifested in the inspection, maintenance, and upgrade of equipment during the operation of the power system. It is the main link to ensure the safe and stable operation of the SG. Consequently, the assessment for management ability of operation and maintenance include the degree of equipment maintenance automation, the ability of precise equipment management, and the flexibility of equipment delivery and configuration.

The degree of equipment maintenance automation is used to measure the automation degree of equipment maintenance in the power system. Relying on the construction of PIoT, the equipment maintenance in the power system is more automated. For example, we use $5 \mathrm{G}$ technology to integrate real time and offline information on grid feeders and users to realize the monitoring, protection, and control of the equipment in power system. The ability of precise equipment management is used to measure the ability of PIoT manage equipment. The flexibility of equipment delivery and configuration can measure the ability of PIoT to provide different service functions according to application scenarios.

2.2. Economic Index. If promoting the construction of PIoT cannot create considerable economic benefits, the technical breakthrough will be meaningless. This paper assesses the economic benefits for the PIoT in integration with other economic indexes such as cost and direct benefits.

All in all, the multidimensional assessment framework of PIoT is shown in Table 1.

\section{Designing Assessment Scheme and Case Study}

\subsection{Using ACKOT to Design PIoT Assessment Scheme under PDS}

3.1.1. ACKOT and Application. The assessment framework for PIoT in Section 1 is a universal assessment template. This template can meet the requirement for the ability assessment of PIoT in power generation, transformation, transmission, and distribution. However, the core abilities of PIoT have different focuses in different PSS. Since different PSS have requirements of common and difference for the core abilities of PIoT, the assessment framework should be personalized according to specific scenarios. Hence, we use ACKOT to reshape the assessment framework under different PSS. The ACKOT aims to dig deeper into the requirement characteristics of different PSS. The general assessment template can be instantiated according to the requirements of PSS. In this way, the index that can measure ability of PIoT to meet the requirements of different PSS can be found.

Assessment template containing commonality and individuality are built for different PSS. Next, the assessment template is applied to the diversified PSS, and an assessment scheme is designed that is compatible with PIoT abilities-PSS requirements. The scheme design process is shown in Figure 1.

Compared with other PSS, the distribution network is closely related to every user and device in every household. Additionally, in the SG, the interdependence between the distribution network and the communication network 
TABLE 1: The multi-dimensional assessment framework of PIoT.

\begin{tabular}{|c|c|c|}
\hline First-level index & Second-level index & Third-level index \\
\hline \multirow{16}{*}{ Technical } & \multirow{6}{*}{ Ubiquitous perception ability } & Terminal deployment coverage \\
\hline & & Rate of successful data collection \\
\hline & & Accuracy of data collection \\
\hline & & Variety of terminal perception frequency \\
\hline & & Diversity of sensing data \\
\hline & & Ability of data sharing \\
\hline & \multirow{4}{*}{ Applications' QoS guarantee } & Reliability for meeting application requirements \\
\hline & & Communication quality of service \\
\hline & & Quality of data processing \\
\hline & & Security and robustness \\
\hline & \multirow{3}{*}{ Security protection ability } & Ability of preventing data leakage \\
\hline & & Ability of anti-interference for communication network \\
\hline & & Ability of resisting natural disasters \\
\hline & \multirow{3}{*}{ Maintenance management ability } & Degree of equipment maintenance automation \\
\hline & & Degree of precise equipment management \\
\hline & & Flexibility of equipment delivery and configuration \\
\hline \multirow{10}{*}{ Economic } & \multirow{3}{*}{ Cost } & Cost of construction \\
\hline & & Cost of maintenance \\
\hline & & Saved investment of PG construction \\
\hline & \multirow[t]{2}{*}{ Direct benefits } & Decreased cost of human resource \\
\hline & & Saved cost of equipment operation \\
\hline & \multirow[b]{2}{*}{ Indirect benefits } & User satisfaction \\
\hline & & Improved quality of power supply \\
\hline & \multirow{3}{*}{ Comprehensive index } & $\begin{array}{l}\text { The level of line loss management in the same period } \\
\text { Payback period }\end{array}$ \\
\hline & & Net present value \\
\hline & & Annual cost \\
\hline
\end{tabular}

makes them inseparable. The distribution network provides energy source for the communication network, and the communication network provides information support for the distribution network, which means they are mutualistic symbiosis. Consequently, the SG in the $5 \mathrm{G}$ era will coruscate more vitality and better promote the interconnection of distribution network and communication network. Thereafter, the construction of a new type of power system is accompanied by the access of a large number of new energy sources with distributed and fragmented, which will pose a greater challenge to the distribution network. Hence, we need to build an assessment scheme of PIoT in the PDS to provide a solid guarantee foundation for the reasonable planning of the distribution network. ACKOT is used to reshape the assessment framework of PIOT in PDS.

3.1.2. Designing the Assessment Scheme of PIoT under the $P D S$. The starting point of the distribution network is connected to transmission lines, and the end point is connected to various power consumers. Hence, if the security and robustness of PIoT in the distribution network is not improved, it will affect the power demand of end users. For example, because of the malfunctions of the aggregation node and the distribution box, the power supply ability of the PG is affected, which will not be able to meet the power demand of the end users. The power consumption information of users is related to the power purchase privacy of end users, and the accuracy of power consumption information also affects the power dispatching planning of distribution network. Thus, the ability of preventing data leakage is used to measure the system's ability to protect the confidentiality and integrity of data information. Moreover, extreme disasters will damage the terminal, which will affect the data collection abilities of the PIoT, and make the PIoT unable to meet the data requirements of the distribution network. Hence, it is necessary to assess the ability of resisting natural disasters of the terminal and strengthen the deployment of disaster prevention facilities in the distribution network.

In the above analysis, first, ACKOT is used to explore the characteristics of the requirement for security protection ability of PIoT in PDS. Next, indexes such as security and robustness, ability of preventing data leakage, and ability of resisting natural disasters are found in the assessment framework. The following will continue to use this technology to explore the remaining requirement characteristics of PIoT and reshape the assessment framework in PDS.

Smart meters are the most typical sensors in power distribution systems. It can collect the user's power consumption data in real time to meet the basic needs of PG companies for power dispatch. The higher the accuracy of collection data, the higher the reference value for PG. Here, we use the accuracy of data collection to measure the similarity between collection data and real data. To prevent accidents, the distribution station must monitor the operation of various equipment as well as collecting user electricity consumption data. Thus, a huge amount of data needs to be collected. The collection of massive data relies on the extensive deployment of terminals. The terminal 


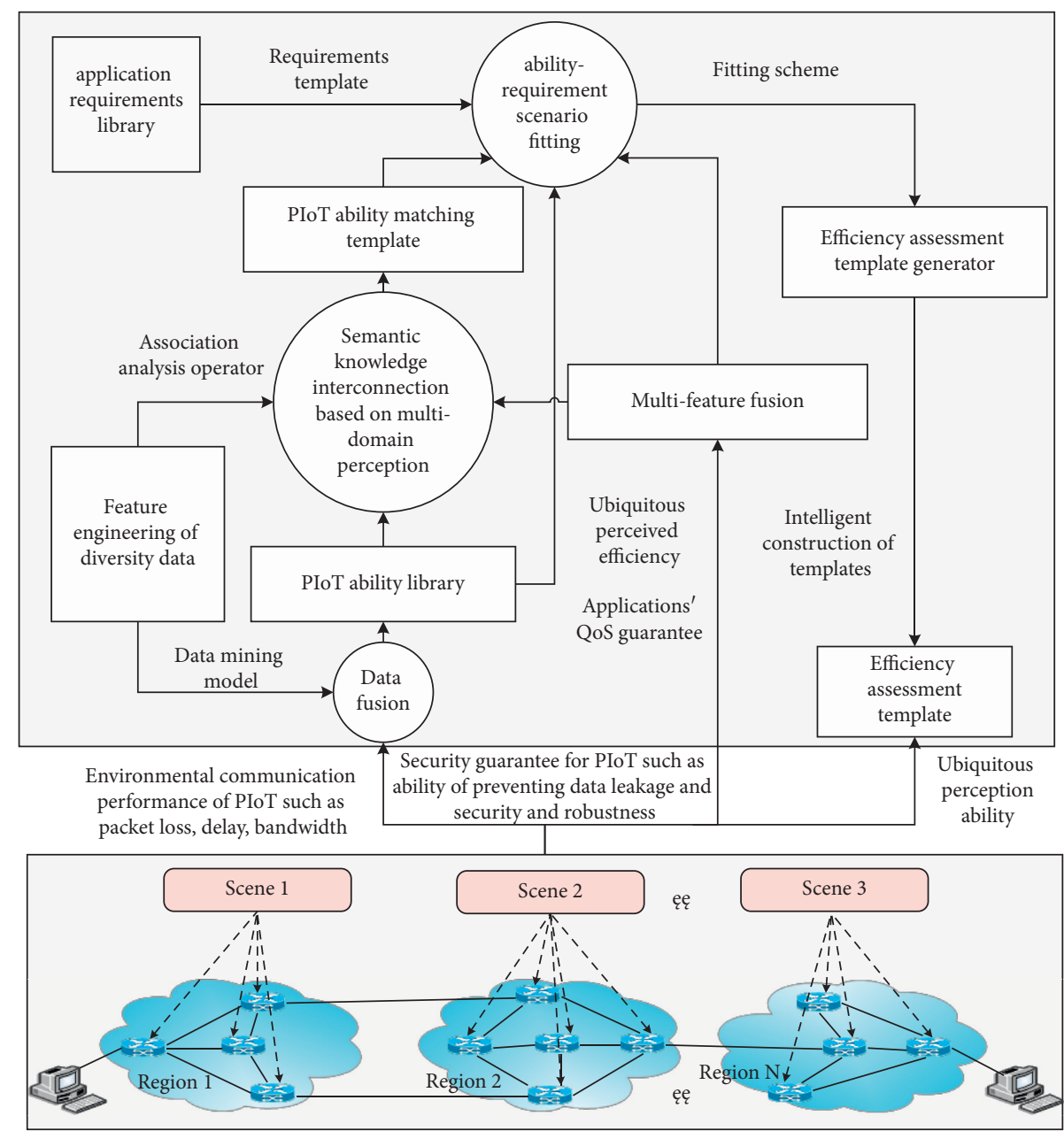

FIGURE 1: The instantiate generation scheme of the assessment framework by using ACKOT. The general assessment framework is instantiated by using this generation scheme according to the requirement of different PDS.

deployment coverage is used to measure the coverage level of the terminal. Different types of data are collected by the terminal. For this reason, the diversity of sensing data and the variety of terminal perception frequency are indispensable indexes to measure the ubiquitous perception ability of the distribution network.

To facilitate data sharing between users and the PG, the distribution network should establish a data sharing mechanism. Consequently, the ability of data sharing can be used to assess the performance of the data sharing mechanism in the distribution network. Reliability for meeting application requirements is used to measure the ability of the terminal to always meet the application requirements of the distribution network, for example, whether the signal and power of the wireless terminal can meet the requirement for the collection and transmission of electricity information. The deep coupling between the distribution network and the communication network makes the communication system of the PIoT very flexible in the PDS. Here, the communication quality of service is used to measure the communication ability of the terminal. Meanwhile, using smart chips will improve the terminal's ability to process massive data. Quality of data processing is used to measure the ability of terminal processing data.

The amount of data in the system needs to be transmitted, stored, and processed rapidly if the number of perceived terminals and users of the distribution network increases in varying degrees. Consequently, the PIoT should have a certain functional extensibility. Based on this, we should improve the flexibility of equipment delivery and configuration to cope with possible changes in work volume in the future. Relying on the construction of PIoT, the management of the distribution station is refined, which can improve the ability of precise equipment management. Furthermore, we can improve the automation level of distribution terminal with improving the degree of equipment maintenance automation. The work efficiency of power distribution equipment will increase with the enhancement of the automation level of distribution terminal.

The improvement of the work efficiency of power distribution equipment can reduce the cost of construction and labor input, which decreased the cost of human resource. The function of the distribution station is to supply power to users within the radiation range and share data with users. Users can interact with the power distribution system in real time through smart terminals in the station area. Thus, users can 
obtain more comprehensive data information according to their own needs to improve user satisfaction. Furthermore, with the rapid development of power industry and energy industry, distributed power supply has become an indispensable and useful supplement to the power supply of PG. The addition of distributed power sources to the distribution network will greatly increase the complexity and uncertainty of the investment in the construction of the distribution system. Thus, reasonable capital planning and allocation is very necessary. The payback period, annual costs, and net present value can be used to assess the scientific planning and precise investment of the distribution network.

In summary, the assessment scheme of PIoT in the PDS is designed, as shown in Figure 2.

\subsection{Comprehensive Assessment Model for PIoT Based on the} Collaboration of PCA and AHP. The assessment scheme built in Section 2.1 has many dimensions. Hence, firstly, we need to simplify the dimension of index before assessing the efficiency of PIoT. Principal component analysis (PCA), as a method to simplify data sets, is often used to reduce the dimension of datasets [30]. Secondly, we need to assign index weights according to the importance of each index. Analytic hierarchy process (AHP) is to compare the importance of indexes in pairs, which is more helpful to the reasonable distribution of index weights [31].

In summary, an assessment model of PIoT based on the collaboration of PCA and AHP is designed. The specific process of the assessment model design is shown in Figure 3. The assessment process of PIoT is summarized as follows:

Step 1: referring to national standards, the PIoT assessment framework is designed according to the principles of index system construction

Step 2: we need to determine the normalization standard and normalize the original measured value

Step 3: using PCA to compute the secondary index value

(1) Normalizing the three-level index.

(2) Computing the eigenvalues and eigenvectors of the index covariance matrix.

(3) Constructing the assessment function: we determine the number of the principal component by setting the variance contribution rate threshold and sorting the cumulative contribution rate or eigenvalues of the principal component. The expression of the comprehensive assessment function is formulated as follows:

$$
f=\alpha_{1} F_{1}+\alpha_{2} F_{2}+\alpha_{3} F_{3}+\cdots+\alpha_{m} F_{m} .
$$

Here, $F_{1}, F_{2}, \ldots, F_{m}$ are the new principal component, and $\alpha_{1}, \alpha_{2}, \ldots, \alpha_{m}$ are the weight of the new principal component.

(4) We can obtain the value of the second-level index if the normalized value of each third-level index is brought into the assessment function obtained in Step 3 , that is, $f_{1}, f_{2}, \ldots, f_{k}$.
Step 4: Computing the assessment value of the PIoT. The mathematical expression of the assessment model of the PIoT is given as follows:

$$
\begin{gathered}
\left\{\begin{array}{l}
V_{1}=\sum_{n=1}^{4} W_{1 n} V_{1 n}, \\
V_{2}=\sum_{m=1}^{4} W_{2 m} V_{2 m},
\end{array}\right. \\
C=W_{1} V_{1}+W_{2} V_{2} .
\end{gathered}
$$

Here, $V_{1}$ and $V_{2}$ represent the value of technical index and economic index, respectively, $W_{11}, W_{12}, \ldots, W_{1 n}$ and $W_{21}, W_{22}, \ldots, W_{2 m}$ represent the weight of each secondary index which belongs to the technical index and economic index, respectively, $V_{11}, V_{12}, \ldots, V_{1 n}$ and $V_{21}, V_{22}, \ldots, V_{2 m}$ represent the value of each secondary index which belongs to the technical index and economic index respectively, $C$ represents the assessment value of PIoT, $W_{1}$ and $W_{2}$ represent the index weight of technical and economic, respectively.

Step 5: Determine the efficiency level of the PIoT.

When computing the assessment value of the PIoT, the range of assessment value and weight is [0-1]. We can determine the efficiency level of the PIoT by Table 2 after computing the assessment value.

3.3. Case Study. Beijing being one of the major cities to promote electric vehicles (EVs), the impact of massive EV access to the distribution network cannot be ignored. To implement the national policy on the development of EV, the Special Plan for Beijing Electric Vehicle Charging Infrastructure (2016-2020) has been put forward in Beijing. According to the plan, about six hundred thousand EVs will be needed in the city by 2021, and about four hundred and thirty-five thousand EV charging piles will be built. The popularization of EV will bring unprecedented impact and challenges to the distribution network. Thus, the assessment of PIoT under the PDS in Beijing is particularly important.

Step 1. Computing the normalized value of the third-level index.

We use charging piles to collect the original values of the three-level index and convert them to [0-1]. The normalized value of the index is shown in Table 3 .

Step 2. Using PCA to compute the secondary index value.

First, we seek the opinions of many experts in the electric power and communication fields anonymously. Next, the expert opinions are counted, processed, analyzed, and summarized. Finally, conduct multiple rounds of consultation, feedback, and adjustment of expert opinions. The scoring results of the experts on the three-level index are shown in Table 4.

The PIoT assessment scheme in PDS is composed of 23 three-level indexes. Firstly, we treat these 23 indexes as 23 random variables, denoted as $x_{1}, x_{2}, \ldots, x_{23}$. Secondly, we 


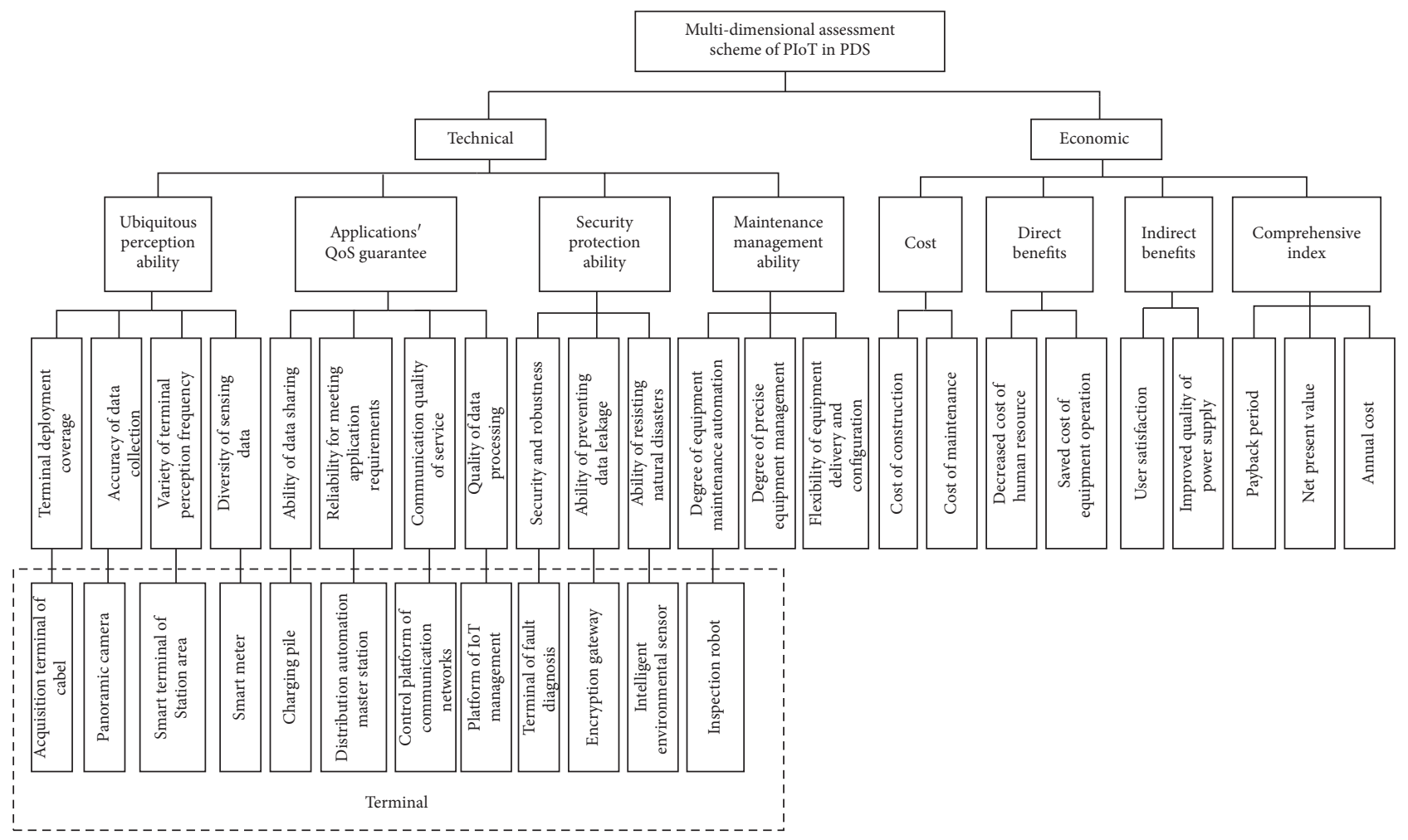

FIgURE 2: The assessment scheme of PIoT in PDS. This assessment scheme can be used as a template for assessing PIoT requirement ability in PDS.

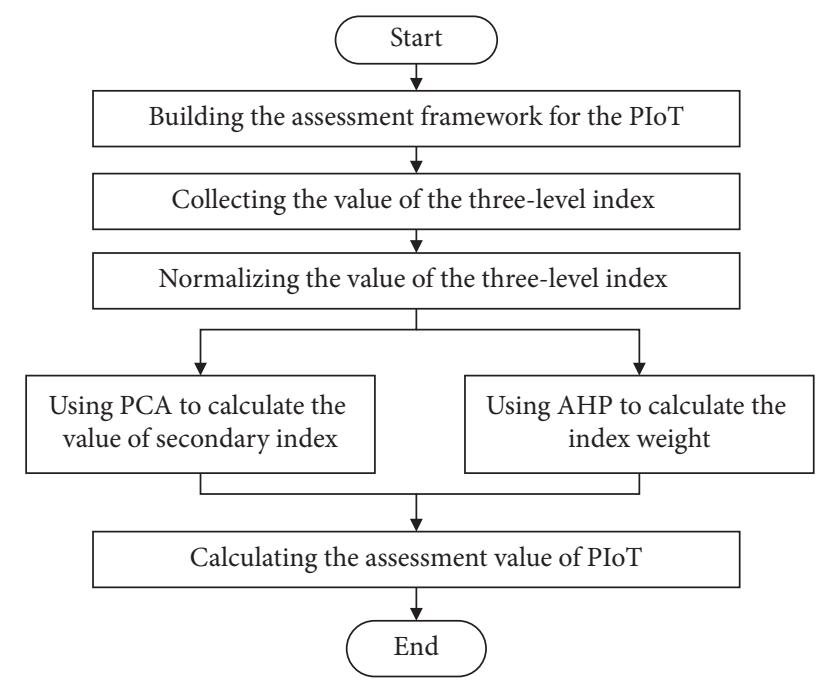

Figure 3: The design process of the assessment model based on the collaboration of PCA and AHP. This assessment model is used to assess the efficiency of PIoT.

TABLE 2: The judgment level for the efficiency of PIoT.

\begin{tabular}{lccccc}
\hline Efficiency level & Danger & Poor & Medium & Good & Excellent \\
\hline Assessment value & $0-0.2$ & $0.2-0.4$ & $0.4-0.6$ & $0.6-0.8$ & $0.8-1$ \\
\hline
\end{tabular}

use PCA to transform these 23-dimensional indexes into linear combinations grouped by the secondary index. Thirdly, computing the eigenvalues and eigenvectors of the corresponding index covariance matrix according to the secondary index grouping, which can obtain the new principal component and its weight. The new principal component and its weight are shown in Table 5. Finally, we get the value of the three-level index to compute the value of the second-level index by formula (1). The value of the secondary index is shown in Table 6 .

Step 3. Computing the index weight based on AHP.

Using AHP to assign weight to index. The weight distribution results are shown in Table 7.

Step 4. Computing the assessment value of the PIoT.

First, combining the value and the weight of the secondary index in Tables 6 and 7, respectively, the value of the technical index and economic index are calculated by using formula (2). Next, combining the weight and the value of the technical index and economic index, the assessment value of the PIoT is calculated by using formula (3).

Step 5. Determining the efficiency level of the PIoT.

According to the computation in Step 4, the assessment value of PIoT is 0.8687 , which belongs to $(0.8,1)$. The assessment level of the PIoT is excellent with reference to Table 2, which further verifies the feasibility and effectiveness of the assessment model.

Step 6. Analyzing the results of PIoT efficiency assessment.

Analyzing the assessment value of PIoT, we found that the values of indirect benefits and comprehensive index are 
TABLE 3: The normalizing value of the three-level index.

\begin{tabular}{lcc}
\hline Secondary index & Three-level index & The value of three-level index \\
\hline & Terminal deployment coverage & 0.89 \\
Ubiquitous perception ability & Accuracy of data collection & 0.86 \\
& Variety of terminal perception frequency & 0.91 \\
& Diversity of sensing data & 0.92 \\
& Ability of data sharing & 0.87 \\
Applications' QoS guarantee & Reliability for meeting application requirements & 0.88 \\
& Communication quality of service & 0.94 \\
Security protection ability & Quality of data processing & 0.90 \\
& Security and robustness & 0.90 \\
Maintenance management ability & Ability of preventing data leakage & 0.92 \\
& Ability of resisting natural disasters & 0.96 \\
Cost & Degree of equipment maintenance automation & 0.91 \\
Degree of precise equipment management & 0.86 \\
Direct benefits & Clexibility of equipment delivery and configuration & 0.85 \\
Indirect benefits & Cost of construction & 0.84 \\
& Decreased cost of human resource & 0.83 \\
Comprehensive index & Saved cost of equipment operation & 0.82 \\
& User satisfaction & 0.88 \\
& Improved quality of power supply & 0.85 \\
& Payback period & 0.86 \\
& Net present value & 0.85
\end{tabular}

TABLE 4: The scoring results of the experts on the three-level index.

\begin{tabular}{|c|c|c|c|c|c|c|c|c|c|c|}
\hline Three-level index & Score 1 & Score 2 & Score 3 & Score 4 & Score 5 & Score 6 & Score 7 & Score 8 & Score 9 & $\begin{array}{c}\text { Score } \\
10\end{array}$ \\
\hline Terminal deployment coverage, $X_{1}$ & 8.8 & 9.0 & 8.6 & 9.1 & 8.7 & 8.8 & 8.6 & 8.7 & 8.9 & 9.0 \\
\hline Accuracy of data collection, $X_{2}$ & 9.0 & 8.7 & 8.7 & 8.6 & 8.5 & 9.1 & 8.6 & 8.7 & 8.2 & 8.4 \\
\hline Variety of terminal perception frequency, $X_{3}$ & 8.7 & 8.9 & 9.0 & 8.7 & 8.7 & 8.7 & 8.9 & 9.1 & 8.6 & 8.8 \\
\hline Diversity of sensing data, $X_{4}$ & 8.9 & 8.7 & 8.9 & 8.3 & 8.5 & 9.0 & 8.4 & 8.5 & 9.1 & 8.7 \\
\hline Ability of data sharing, $X_{5}$ & 8.4 & 8.6 & 8.7 & 8.5 & 9.0 & 8.7 & 8.6 & 8.8 & 8.5 & 9.0 \\
\hline $\begin{array}{l}\text { Reliability for meeting application } \\
\text { requirements, } X_{6}\end{array}$ & 8.6 & 9.0 & 8.8 & 8.7 & 9.1 & 8.7 & 8.9 & 8.6 & 9.1 & 8.9 \\
\hline Communication quality of service, $X_{7}$ & 9.1 & 9.3 & 8.7 & 8.9 & 7.7 & 8.7 & 8.4 & 8.6 & 9.0 & 8.5 \\
\hline Quality of data processing, $X_{8}$ & 8.9 & 8.8 & 9.0 & 8.6 & 8.4 & 8.7 & 8.6 & 8.4 & 9.1 & 8.9 \\
\hline Security and robustness, $X_{9}$ & 9.2 & 8.9 & 8.7 & 9.0 & 8.8 & 8.8 & 9.2 & 8.9 & 9.0 & 8.7 \\
\hline Ability of preventing data leakage, $X_{10}$ & 8.9 & 8.7 & 9.1 & 8.6 & 8.7 & 9.0 & 8.7 & 8.8 & 8.6 & 8.4 \\
\hline Ability of resisting natural disasters, $X_{11}$ & 9.0 & 8.8 & 8.7 & 8.9 & 9.2 & 9.1 & 8.8 & 9.0 & 9.2 & 8.7 \\
\hline $\begin{array}{l}\text { Degree of equipment maintenance automation, } \\
X_{12}\end{array}$ & 8.4 & 9.0 & 8.6 & 8.4 & 8.3 & 8.3 & 8.4 & 8.7 & 8.4 & 8.4 \\
\hline Degree of precise equipment management, $X_{13}$ & 8.7 & 8.6 & 8.3 & 8.9 & 8.9 & 8.7 & 8.6 & 8.4 & 8.5 & 8.7 \\
\hline $\begin{array}{l}\text { Flexibility of equipment delivery and } \\
\text { configuration, } X_{14}\end{array}$ & 8.5 & 8.7 & 8.4 & 8.6 & 8.5 & 8.32 & 8.7 & 8.4 & 8.5 & 8.7 \\
\hline Cost of construction, $X_{15}$ & 8.8 & 9.3 & 8.9 & 8.6 & 9.0 & 8.7 & 9.0 & 8.5 & 8.3 & 9.0 \\
\hline Cost of maintenance, $X_{16}$ & 9.0 & 9.5 & 9.0 & 8.9 & 8.8 & 8.9 & 8.6 & 8.8 & 8.6 & 8.8 \\
\hline Decreased cost of human resource, $X_{17}$ & 9.2 & 9.3 & 8.9 & 9.1 & 9.2 & 8.9 & 9.0 & 8.7 & 8.9 & 9.0 \\
\hline Saved cost of equipment operation, $X_{18}$ & 8.8 & 8.8 & 9.0 & 9.1 & 9.0 & 8.6 & 8.8 & 9.0 & 8.7 & 8.6 \\
\hline User satisfaction, $X_{19}$ & 9.1 & 9.1 & 9.0 & 9.0 & 8.7 & 8.8 & 8.9 & 8.7 & 9.2 & 9.0 \\
\hline Improved quality of power supply, $X_{20}$ & 9.0 & 9.2 & 9.2 & 9.4 & 8.8 & 9.0 & 8.8 & 9.1 & 9.0 & 9.3 \\
\hline Payback period, $X_{21}$ & 9.2 & 9.0 & 8.8 & 9.1 & 9.1 & 8.9 & 8.9 & 8.7 & 8.9 & 9.0 \\
\hline Net present value, $X_{22}$ & 8.6 & 8.4 & 8.3 & 8.4 & 8.5 & 8.1 & 8.3 & 8.2 & 8.3 & 8.5 \\
\hline Annual cost, $X_{23}$ & 8.5 & 8.6 & 8.4 & 8.5 & 8.7 & 8.2 & 8.4 & 8.4 & 8.5 & 8.6 \\
\hline
\end{tabular}

low as seen in Table 6. Due to the generalization of the secondary index, it is impossible to give specific reference suggestions for the improvement and optimization of the PIoT. Consequently, we refine the result analysis to the third-level index, such as user satisfaction and payback period.

As one of the major cities that vigorously promote $\mathrm{EV}$, the penetration rate of $\mathrm{EV}$ is increasing year by year in an 
TABLE 5: The weight of new principal components.

\begin{tabular}{lcc}
\hline Secondary index & New principal components & Weight \\
\hline Ubiquitous perception ability & $F_{1}$ & 0.80683 \\
Applications' QoS guarantee & $F_{2}$ & 0.60520 \\
Security protection ability & $F_{3}$ & 0.32156 \\
Maintenance management ability & $F_{4}$ & 0.84632 \\
Cost & $F_{5}$ & 0.572306 \\
Direct benefits & $F_{6}$ & 0.30843 \\
Indirect benefits & $F_{7}$ & 0.79049 \\
Comprehensive index & $F_{8}$ \\
\hline
\end{tabular}

Table 6: The value of secondary index by using PCA.

\begin{tabular}{lr}
\hline Secondary index & Value \\
\hline Ubiquitous perception ability & 0.9154 \\
Applications' QoS guarantee & 0.9512 \\
Security protection ability & 0.9397 \\
Maintenance management ability & 0.8991 \\
Cost & 0.8508 \\
Direct benefits & 0.7816 \\
Indirect benefits & 0.7007 \\
Comprehensive index & 0.6402 \\
\hline
\end{tabular}

TABLE 7: The distribution result of index weight.

\begin{tabular}{lccc}
\hline First-level index & First-level index weight & Secondary index & Secondary index weight \\
\hline & & Ubiquitous perception ability & 0.27 \\
Technical & 0.67 & Applications' QoS guarantee & 0.28 \\
& & Security protection ability & 0.19 \\
& & Maintenance management ability & 0.26 \\
& \multirow{2}{*}{0.33} & Cost & 0.25 \\
Economic & & Direct benefits & 0.3 \\
& & Indirect benefits & 0.29 \\
& & Comprehensive index & 0.16 \\
\hline
\end{tabular}

exponential manner. The access of large-scale EV has brought profound changes to the scale and structure of the distribution network. Among them, the problem of a sharp increase in power load is more prominent. The insufficiency of power supply during the peak load period will force users to change their inherent power consumption habits, which greatly affects user satisfaction. Meanwhile, because of the disorderly charging of massive EV, it will cause the problem of instability for the voltage and frequency in the distribution network. It will not only affect the improved quality of power supply of the distribution network but also further increase the operating cost of the distribution network. The increase in the operating cost of the distribution network will indirectly affect its payback period and annual cost.

To address the above problems, we can combine demand response strategies to rationally regulate the charging and discharging behavior of EV. As a mobile energy storage device, EV can be charged to absorb the excess wind and light energy during the low load period. Meanwhile, EV can alleviate the power supply pressure of the distribution network by feeding power to the grid during peak load periods. It can effectively avoid problems such as voltage drops caused by insufficient power supply and further improve the power supply quality of the distribution network. Additionally, users can also reasonably arrange their own charging and discharging behaviors through demand response strategies to obtain corresponding benefits, which greatly improves user satisfaction.

\section{Optimization Analysis of the Assessment Model Based on MLT}

The dimensionality of the instantiation assessment scheme of PIoT in Section 2 is high, which causes the overlapping of information among various indexes. Consequently, we assess the efficiency of PIoT with increasing the workload of data collection and processing, which is not conducive to quickly and effectively assessing the efficiency of PIoT.

With the in-depth development of SG, data mining techniques represented by machine learning will be widely used in the field of PG. Among them, dimension reduction 
technology is used to extract the main features of data to reduce the data dimension.

To address the above problems, this section will use MLT to optimize the assessment model. We will use this technology to mine the core information in the assessment scheme and remove the redundant information in the massive index, which reduces the dimensionality of the assessment scheme from high dimension to low dimension. It can reduce the workload of index information collection and processing with reducing the dimensionality of the assessment scheme, which will improve the timeliness of assessment.

We tried several machine learning methods such as decision tree (DT), extra tree and AdaBoost to achieve dimensionality reduction, among which the effect of DT is better. Compared with other machine learning methods, DT has the following advantages: (1) it can deal with numerical data and classified data. (2) It uses the white box model. (3) The reliability of the model can be verified by numerical statistical tests. In summary, the DT model is selected for classification training of sample data under the environment of Python 3.8.1. Next, using classification accuracy to extract certain features that play a key role in classification decisionmaking. The optimization process of the assessment model based on MLT is shown in Figure 4.

Here, the efficiency of PIoT under the PDS in Beijing is assessed by the optimization process of the assessment model.

Step 1: building a sample data set.

A sample data set of the distribution station in Beijing is randomly selected, as shown in Table 8 . The data set of the experiment in this section consists 100 such data set.

Step 2: optimizing the application of the assessment model.

Using the DT to classify and train the data set, we can get the index importance of value and ranking, as shown in Figure 5. To make Figure 5 have a better display effect, we use the item number in Table 8 as a symbol for the index.

As shown in Figure 5, the importance values of the first four indexes are relatively large, and the other indexes are 0 . Hence, we select the important values of these four indexes as the index weight after dimensionality reduction, as shown in Table 9.

The dimensionality of the assessment model is reduced by using DT, in which complexity of the algorithm is $O\left(n k \log _{2} n\right)$ [32]. Here, $n$ and $k$ are the number of samples in the training set and the dimensionality of the data, respectively. In this simulation experiment, $n=100, k=23$. Consequently, the complexity of the proposed algorithm is $O\left(2.3 * 10^{3} \log _{2} 100\right)$, with the execution time of $1.61 \mathrm{~s}$.

Step 3: Validating the optimization assessment model. Using the DT model to classify and train the dimensionality-reduced index, the classification accuracy rate

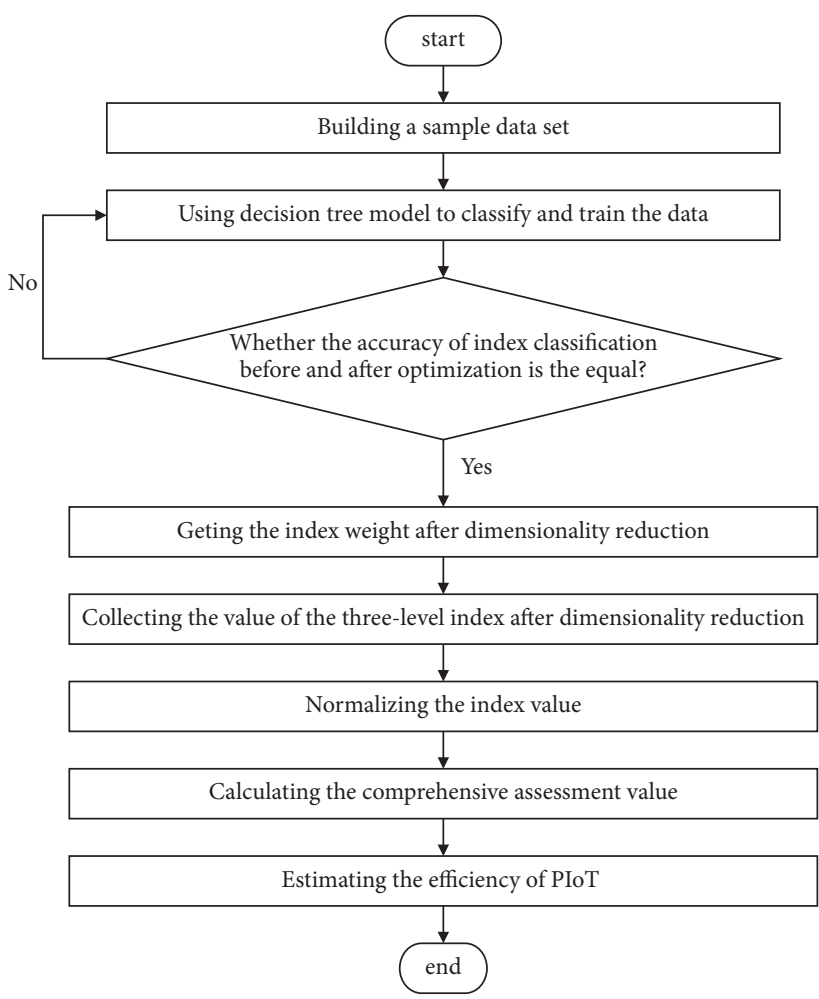

Figure 4: The optimization process of the assessment model based on MLT. This model is used to reduce the dimensionality of the PIoT assessment scheme, which can improve the timeliness of the assessment.

is $83 \%$, which is the same as the 23 -dimensional threelevel indexes in Section 3. That is, the dimensionalityreduced indexes as a new data set can be used to assess the efficiency of PIoT, which can verify the effectiveness of the optimization assessment model.

The performance comparison experiment of the algorithm is designed. The comparison of the proposed algorithm with the existing algorithm $[33,34]$ is shown in Table 10. The results show that three algorithms can achieve the dimensionality reduction of the assessment scheme. Among them, the DT can reduce the assessment scheme with 3-4 dimension with the classification accuracy rate being $83 \%$. The comprehensive performance of the DT is the best by analyzing the results.

Step 4: Evaluating the efficiency of PIoT.

Combining the normalized values of the index in Table 3 and the weight of the four indexes in Table 9 and computing the assessment value of the PIoT by using formula (3) is 0.88474 . Comparing this value to Table 2, we can get the efficiency level of PIoT as excellent. The assessment result is the same as the assessment case in Section 3, which verifies the correctness of the optimization model. Furthermore, when evaluating PIoT, using four indexes as a new data set to replace the original 23 index, it improves the assessment efficiency by $82.6 \%$. It can verify the timeliness of the optimization model in this article. 
TABLE 8: A sample data of the distribution station in Beijing.

\begin{tabular}{|c|c|c|c|}
\hline \multirow{2}{*}{ Second-level index } & \multicolumn{2}{|r|}{ Three-level index } & \multirow{2}{*}{ The value of three-level index } \\
\hline & Item no. & Item definition and meanings & \\
\hline \multirow{4}{*}{ Ubiquitous perception ability } & 1 & Terminal deployment coverage & 0.921321 \\
\hline & 2 & Accuracy of data collection & 0.976143 \\
\hline & 3 & Variety of terminal perception frequency & 0.952878 \\
\hline & 4 & Diversity of sensing data & 0.886099 \\
\hline \multirow{4}{*}{ Applications' QoS guarantee } & 5 & Ability of data sharing & 0.874552 \\
\hline & 6 & Reliability for meeting application requirements & 0.911812 \\
\hline & 7 & Communication quality of service & 0.871818 \\
\hline & 8 & Quality of data processing & 0.942396 \\
\hline \multirow{3}{*}{ Security protection ability } & 9 & Security and robustness & 0.971341 \\
\hline & 10 & Ability of preventing data leakage & 0.925245 \\
\hline & 11 & Ability of resisting natural disasters & 0.967985 \\
\hline \multirow{3}{*}{ Maintenance management ability } & 12 & Degree of equipment maintenance automation & 0.953568 \\
\hline & 13 & Degree of precise equipment management & 0.978742 \\
\hline & 14 & Flexibility of equipment delivery and configuration & 0.979623 \\
\hline \multirow{2}{*}{ Cost } & 15 & Cost of construction & 0.954032 \\
\hline & 16 & Cost of maintenance & 0.887993 \\
\hline \multirow{2}{*}{ Direct benefits } & 17 & Decreased cost of human resource & 0.956793 \\
\hline & 18 & Saved cost of equipment operation & 0.866593 \\
\hline \multirow{2}{*}{ Indirect benefits } & 19 & User satisfaction & 0.921458 \\
\hline & 20 & Improved quality of power supply & 0.923697 \\
\hline \multirow{3}{*}{ Comprehensive index } & 21 & Payback period & 0.875962 \\
\hline & 22 & Net present value & 0.917689 \\
\hline & 23 & Annual cost & 0.915398 \\
\hline
\end{tabular}

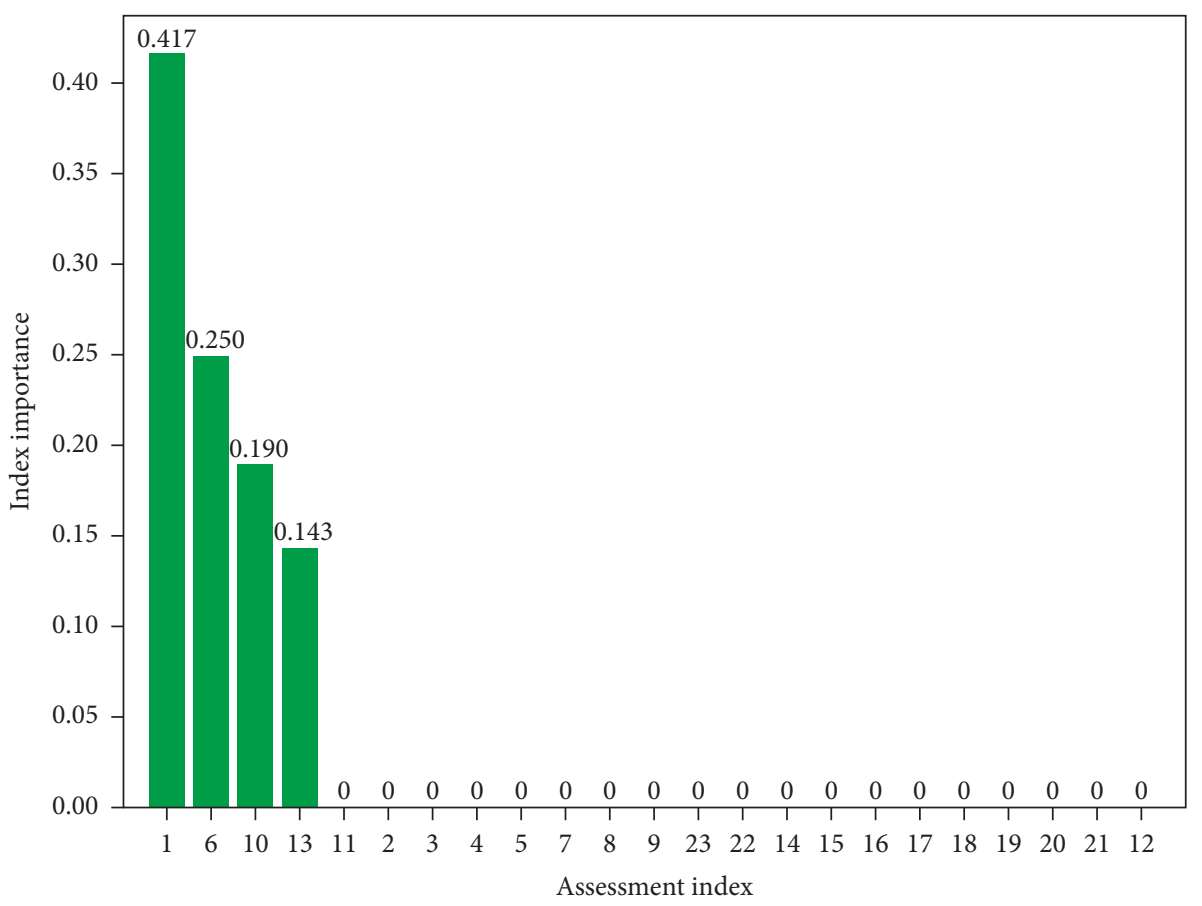

FIgURe 5: The index importance ranking.

TABLE 9: The weight of the index after dimensionality reduction.

\begin{tabular}{lcccc}
\hline $\begin{array}{l}\text { Index after dimensionality } \\
\text { reduction }\end{array}$ & $\begin{array}{c}\text { Terminal deployment } \\
\text { coverage }\end{array}$ & $\begin{array}{c}\text { Reliability for meeting } \\
\text { application requirements }\end{array}$ & $\begin{array}{c}\text { Ability of preventing } \\
\text { data leakage }\end{array}$ & $\begin{array}{c}\text { Degree of precise } \\
\text { equipment management }\end{array}$ \\
\hline Weight & 0.417 & 0.250 & 0.190 & 0.143 \\
\hline
\end{tabular}


TABLE 10: The comparison of the algorithm performance.

\begin{tabular}{lccc}
\hline Algorithm & The dimension of indexes after dimensionality reduction & Classification accuracy rate & The execution time $(\mathrm{s})$ \\
\hline Decision tree & 4 & 0.83 & 1.61 \\
Extra tree & 14 & 0.64 & 1.22 \\
AdaBoost & 4 & 0.42 & 1.19 \\
\hline
\end{tabular}

To avoid random situations in the simulation process, the model is used to conduct an assessment experiment on 1,000 communities with shared charging piles in Haidian district, Beijing. The results of multiple simulation experiments are summed and analyzed. Firstly, we use charging piles to collect the data. Secondly, the collaborative assessment model of PCA and AHP is used to assess the community. Thirdly, the optimization assessment model with using MLT is used to assess the community. Finally, we compare the level of these 1000 cells before and after optimization. Among them, 985 communities have the same grade, and the other 15 communities have different degrees before and after optimization. The difference is shown in the following situation: the ranks of the 9 cells before optimization are excellent as well as after optimization are good. The grade of the 6 cells before optimization is medium as well as that after optimization is good. In summary, the accuracy rate of the optimization assessment model is $98.5 \%$, which verifies the accuracy of the model.

\section{Conclusions}

As the nerve ending of the SG, the PIoT will directly affect the holographic perception capability of the SG. Consequently, firstly, the assessment framework is designed to provide an assessment template for the efficiency assessment of PIoT. Secondly, the needs of different PSS have their own focus. Since the distribution network is the backbone of the power system, an assessment scheme covering the demand characteristics of PDS has been constructed. Finally, we build a collaborative assessment model of PCA and AHP to assess the efficiency of PIoT. On this basis, MLT is used to reduce the dimensionality of the assessment scheme, which improves the timeliness of assessment.

\section{Data Availability}

The data used to support the findings of this study are included within the article. More underlying data involve the privacy of enterprises and users and therefore cannot be published.

\section{Conflicts of Interest}

The authors declare that they have no conflicts of interest regarding the publication of this paper.

\section{Acknowledgments}

This work was supported by the National Natural Science Foundation of China (nos. 61941113 and 61971033) and State Grid Science and Technology Research Program (no. 202056177A).

\section{References}

[1] J. B. Guo, "Scientific and technological innovation supports the construction of new power systems," 2021, http://www. chinasmartgrid.com.cn/news/20210519/638624.shtml.

[2] J. C. Liu, Y. H. Li, Y. Y. Lu, F. Xiaoxu, and Y. Suli, "Research on the influence factors of ubiquitous power internet of things for promoting consumption of wind power based on fuzzy G1-ISM in China," Electrical Power and Energy Systems, vol. 121, pp. 106-124, 2020.

[3] C. S. Guo, F. J. Luo, Z. X. Cai, Y. Zhao, and D. R. Zhangc, "Integrated planning of internet data centers and battery energy storage systems in smart grids," Applied Energy, vol. 281, pp. 1-9, 2021.

[4] A. Zanella, N. Bui, A. Castellani, V. Lorenzo, and Z. Michele, "Internet of Things for smart cities," IEEE Internet of Things Journal, vol. 1, no. 1, pp. 22-32, 2014.

[5] X. Liu and X. Y. Zhang, "NOMA-based resource allocation for cluster-based cognitive industrial Internet of Things," IEEE Transactions on Industrial Informatics, vol. 16, no. 8, pp. 5379-5388, 2020.

[6] X. Liu, M. Umair, X. B. Zhai, W. D. Lu, and C. M. Wu, "QoSguarantee resource allocation for multibeam satellite industrial internet of things with NOMA," IEEE Transactions on Industrial Informatics, vol. 17, no. 3, pp. 2052-2061, 2021.

[7] J. S. Tao, M. Umair, M. Ali, and J. Zhou, "The impact of Internet of Things supported by emerging $5 \mathrm{G}$ in power systems: a review," CSEE Journal of Power and Energy Systems, vol. 6, no. 2, pp. 344-352, 2020.

[8] G. Bedi, G. K. Venayagamoorthy, R. Singh, R. R. Brooks, and K.-C. Wang, "Review of Internet of Things (IoT) in electric power and energy systems," IEEE Internet of Things Journal, vol. 5, no. 2, pp. 847-870, 2018.

[9] X. Y. Kong, F. Y. Sun, X. X. Huo, X. Li, and Y. Shen, "Hierarchical optimal scheduling method of heat-electricity integrated energy system based on Power Internet of Things," Energy, vol. 210, pp. 1-14, 2020.

[10] X. Liu and X. Y. Zhang, "Rate and energy efficiency improvements for 5G-based IoT with simultaneous transfer," IEEE Internet of Things Journal, vol. 6, no. 4, pp. 5971-5980, 2019.

[11] K. H. Wu, J. Y. Wang, W. Li, and Y. Zhu, "Research on the operation mode of new generation electric power system for the future energy internet," Proceedings of the Chinese Society for Electrical Engineering, vol. 39, no. 4, pp. 966-979, 2019.

[12] H. A. Khattak, M. A. Shah, S. Khan, I. Ali, and M. Imran, "Perception layer security in internet of things," Future Generation Computer Systems, vol. 100, pp. 144-164, 2019.

[13] T. Fei, Z. Ying, D. X. Li, and Z. Lin, "IoT-based intelligent perception and access of manufacturing resource toward cloud manufacturing," IEEE Transactions on Industrial Informatics, vol. 10, no. 2, pp. 1547-1557, 2014.

[14] M. H. Ullah and J. D. Park, "Distributed energy trading in smart grid over directed communication network," IEEE Transactions on Smart Grid, vol. 12, no. 4, pp. 3669-3672, 2021.

[15] G. Y. Qin, M. J. Zhang, Q. Y. Yan, C. Xu, and C. M. Kammen, "Comprehensive evaluation of regional energy internet using 
a fuzzy analytic hierarchy process based on cloud model: a case in China," Energy, vol. 228, pp. 1-13, 2021.

[16] M. Masera, E. F. Bompard, and F. Profumo, "Smart (electricity) grids for smart cities: assessing roles and societal impacts," Proceedings of the IEEE, vol. 106, no. 4, pp. 613-625, 2018.

[17] C. S. Lai and G. Locatelli, "Valuing the option to prototype: a case study with generation integrated energy storage," Energy, vol. 217, pp. 1-15, 2021.

[18] Y. M. Liu, J. H. Zhang, X. P. Yang, S. Niu, S. Zheng, and A. Xue, "Construction method of evaluation index system of relay protection based on macroscopic and microcosmic view," Power System Technology, vol. 44, no. 8, pp. 3090-3096, 2020.

[19] K. Yuan, J. R. Li, Y. Song, Y. Mu, and C. Sun, "Review and prospect of comprehensive evaluation technology of regional energy internet," Automation of Electric Power Systems, vol. 43, no. 14, pp. 41-52+64, 2019.

[20] Z. Y. Li, A Study on Economic Efficacy Evaluation of Smart Grid Based on Improved TOPSIS Method, North China Electric Power University, Beijing, China, 2016.

[21] S. Hall and T. J. Foxon, "Values in the smart grid: the coevolving political economy of smart distribution," Energy Policy, vol. 74, pp. 600-609, 2014.

[22] B. Zeng, J. M. Bai, W. Z. Guo, Y. Q. Yang, and M. Zeng, "Comprehensive evaluation for benefits of demand response in smart distribution networks," Power System Technology, vol. 41, no. 5, pp. 1603-1611, 2017.

[23] Y. M. Li and Z. Chen, "Study on regional electric energy substitution potential evaluation based on TOPSIS method of optimized connection degree," Power System Technology, vol. 43, no. 2, pp. 687-693, 2017.

[24] Y. J. Fang, X. L. Wang, J. Shi, X. Wu, and H. Yan, "Research on operation and economic efficiency evaluation of provincial power grid with integrated renewable energy," Power System Technology, vol. 41, no. 7, pp. 2138-2145, 2017.

[25] Z. M. Lu, Y. Gao, and C. B. Xu, "Evaluation of energy management system for regional integrated energy system under interval type-2 hesitant fuzzy environment," Energy, vol. 222, pp. 1-19, 2021.

[26] H. Liu, Y. Zhao, C. H. Gu, S. Ge, and Z. Yang, "Adjustable capability of the distributed energy system: definition, framework, and evaluation model," Energy, vol. 222, pp. 1-17, 2021.

[27] State administration for market regulation, standardization administration, Internet of Things-General Principles of Stipulation on Evaluation Indicator System: GB/T 36468-2018, Standards Press of China, Beijing, China, 2018.

[28] State administration for market regulation, standardization administration, Information Security Technology-Security Technical Requirements of Data Transmission for Internet of Things: GB/T37025-2018, Standards Press of China, Beijing, China, 2018.

[29] General Administration of Quality Supervision, Inspection and Quarantine of the People's Republic of China, Standardization Administration. Information Security TechnologyDisaster Recovery Specifications for Information Systems: GB/T 20988-2007, Standards Press of China, Beijing, China, 2007.

[30] Z. H. Yu and W. L. Chin, "Blind false data injection attack using PCA approximation method in smart grid," IEEE Transactions on Smart Grid, vol. 6, no. 3, pp. 1219-1226, 2015.

[31] X. W. Lin, Research on Comprehensive Evaluation of Smart City Development Based on PSF Mode, Harbin Institute of Technology, Harbin, China, 2017.
[32] X. W. Wang and F. Liu, "Data-driven relay selection for physical-layer security: a decision tree approach," IEEE Access, vol. 8, pp. 12105-12116, 2020.

[33] A. Samat, C. Persello, S. Liu, E. Li, Z. Miao, and J. Abuduwaili, "Classification of VHR multispectral images using Extra Trees and maximally stable extremal region-guided morphological profile," IEEE Journal of Selected Topics in Applied Earth Observations and Remote Sensing, vol. 11, no. 9, pp. 31793195, 2018.

[34] J. Nie, S. C. Yang, Q. Ren, and D. Su, “A Novel Classification Method Based on Adaboost for Electromagnetic Emission Characteristics," in Proceedings of the IEEE International Applied Computational Electromagnetics Society SymposiumChina (ACES), IEEE, Beijing, China, August 2018. 